\title{
MicroRNA-616 promotes the progression of ovarian cancer by targeting TIMP2
}

\author{
ZHONGBO CHEN, JIANQING ZHU, YIMING ZHU and JUNJIAN WANG \\ Department of Gynecologic Oncology, Zhejiang Cancer Hospital, Hangzhou, Zhejiang 310022, P.R. China
}

Received October 14, 2017; Accepted April 3, 2018

DOI: $10.3892 /$ or.2018.6368

\begin{abstract}
MicroRNAs (miRNAs), a group of short ( 20 nt) non-coding RNAs, play critical roles in the development and progression of ovarian cancer (OC). The role of miR-616, a recently identified cancer-associated miRNA, has never been examined in OC before. The present study demonstrated that the level of miR-616 was increased in OC tissues. A high miR-616 level was associated with poor tumor differentiation and advanced tumor-node-metastasis (TNM) stage. Survival analysis revealed that an elevated level of miR-616 was associated with poor prognosis of OC patients as demonstrated by decreased overall survival (OS) and disease-free survival (DFS). Overexpression of miR-616 promoted the migration, invasion as well as epithelial-mesenchymal transition (EMT) of A2780 cells. Knockdown of miR-616 inhibited these biological functions. Immunohistochemical (IHC) staining revealed that OC tissues with high miR-616 levels exhibited a significantly decreased level of E-cadherin and an increased level of N-cadherin. Furthermore, tissue inhibitor of metalloproteinases 2 (TIMP2) was confirmed to be a direct downstream target of miR-616. Inhibition of TIMP2 expression was required for the promoting effects of miR-616 on the metastasis and EMT of OC cells. Collectively, this study revealed that miR-616 promoted the progression of OC by enhancing cell migration, invasion and EMT.
\end{abstract}

\section{Introduction}

Ovarian cancer (OC) is a fatal gynecologic cancer worldwide accounting for most cancer-associated mortalities in women with gynecologic cancer (1). Most patients diagnosed with OC are at advanced stages, leading to unavailability of curative treatments. Therefore, the long-term prognosis of OC patients is poor, with a low 5-year overall survival rate of $\sim 40 \%$ (2). Therefore, investigating the molecular mechanisms underlying

Correspondence to: Dr Zhongbo Chen, Department of Gynecologic Oncology, Zhejiang Cancer Hospital, 38 Guangji Road, Hangzhou, Zhejiang 310022, P.R. China

E-mail: gezellig@163.com

Key words: miR-616, ovarian cancer, TIMP2, metastasis, epithelialmesenchymal transition the progression of OC may facilitate the discovery of novel biomarkers and therapeutic targets of OC.

MicroRNAs (miRNAs), a group of small non-coding RNAs with $\sim 20$ nucleotides (3), can regulate the expression of target genes in a post-transcriptional manner by interacting with the 3'-untranslated region (3'-UTR) of mRNAs (4). miRNAs play versatile roles in development and disease processes (5). miRNAs have been found to play critical roles in various human cancers $(6,7)$. In OC, miRNAs can potentially serve as biomarkers and therapeutic targets for OC patients (8). However, the role of specific miRNAs in OC remain to be investigated. Recently, miR-616 was identified to be a cancer-associated miRNA. It promoted the migration, invasion and epithelialmesenchymal transition (EMT) of hepatocellular carcinoma through inhibition of PTEN expression (9). In prostate cancer, miR-616 promoted the growth of prostate cancer by inhibiting tissue factor pathway inhibitor TFPI-2 (10). In lung cancer, sulforaphane suppressed the EMT and metastasis of lung cancer cells through the miR-616-mediated GSK3 $\beta / \beta$-catenin signaling pathways (11). However, the role of miR-616 in OC and the underlying molecular mechanisms remain unknown.

In the present study, we demonstrated that the level of miR-616 in OC was elevated. Compared with patients without metastasis, OC patients with metastasis had a significantly increased miR-616 level. An increased level of miR-616 was associated with poor tumor differentiation, advanced tumornode-metastasis (TNM) stages and poor prognosis of OC patients. Functionally, miR-616 enhanced the ability of the migration, invasion and EMT of OC cells. Furthermore, our data revealed that miR-616 interacted with TIMP2 3'-UTR and inhibited the expression of TIMP2. TIMP2 overexpression inhibited the promoting effects of miR-616 overexpression on cell metastasis and EMT while knockdown of TIMP2 reversed the inhibitory effects of miR-616 knockdown on these cellular functions. Collectively, this study demonstrated that miR-616 is an oncogenic miRNA in OC and promotes the progression of OC by enhancing cell metastasis and EMT.

\section{Materials and methods}

Clinical samples and cell culture. Sixty pairs of OC samples and non-tumor tissues were collected from the Department of Gynecologic Oncology, Zhejiang Cancer Hospital between January 2008 to December 2011. Only patients with complete information of clinical features and complete survival 
information were included. All clinical tissues collected in this study were pathologically confirmed as OC. Informed consent was obtained from every patient enrolled in this study. The protocol of this study was approved by the Institutional Research Ethics Committee of Zhejiang Cancer Hospital.

TheFTE187 cell line, an immortalized human fallopian tube epithelial cell line and five types of human OC cells (A2780, CAOV3, HO-8910, SKOV-3 and ES-2) were purchased from the American Type Culture Collection (ATCC; Manassas, VA, USA). Dulbecco's modified Eagle's medium (DMEM) supplemented with fetal bovine serum (FBS) (both from Gibco; Thermo Fisher Scientific, Inc., Waltham, MA, USA), penicillin and streptomycin, were used for all cell cultures of OC cells. Medium 199 and MCDB105 medium mixed at a ratio of 1:1 supplemented with FBS and EGF (all from Sigma-Aldrich; Merck KGaA, Darmstadt, Germany), were used for the culture of FTE187 cells. Cell cultures were maintained in a humidified incubator with $5 \% \mathrm{CO}_{2}$ at $37^{\circ} \mathrm{C}$.

Cell transfection. miR-616 mimics, miR-616 inhibitors, control mimics and negative control inhibitors were obtained from Guangzhou GeneCopoeia (Guangzhou, China). TIMP2 and the control vector, as well as TIMP2 siRNA and negative control siRNA were purchased from Addgene (Cambridge, MA, USA). All vectors or siRNAs were transfected into OC cells using Lipofectamine $^{\circledR} 2000$ (Invitrogen, Carlsbad, CA, USA).

Real-time quantitative reverse transcription-PCR ( $q R T-P C R)$. TRIzol was used to extract the RNA from clinical samples and OC cells. qRT-PCR assays in the present study were performed using a miRNA Reverse Transcription kit and a Human miRNA assay kit (both from Applied Biosystems; Thermo Fisher Scientific, Inc.). Primers for miR-616, TIMP2, GAPDH and U6 were purchased from GeneCopoeia. U6 and GAPDH were used as internal controls for miR-616 and TIMP2, respectively. The primers sequences were as follows: miR-616 forward, 5'-CTGTGTGCCACACAGTTTG-3' and reverse, 5'-CGGC CCTTAACTCATTCTTT-3'; U6 forward, 5'-CGCTTCGGC AGCACATATAC-3' and reverse, 5'-CAGGGGCCATGCT AATCTT-3'; TIMP2 forward, 5'-CTCGGCAGTGTGTGGG GTC-3' and reverse, 5'-CGAGAAACTCCTGCTTGGGG-3'; and GAPDH forward, 5'-ATTCCATGGCACCGTCAAGGC TGA-3' and reverse, 5'-TTCTCCATGGTGGTGAAGACG CCA-3'.

Western blotting. Cellular protein was extracted using RIPA lysis buffer, and a BCA kit (Pierce; Thermo Fisher Scientific, Inc.) was employed for the measurement of protein concentration. After being loaded and separated on 4-20\% SDS gels, cellular proteins were transferred to polyvinylidene fluoride (PVDF) membranes. The membranes were incubated with the primary antibodies. Primary antibodies used in this study included TIMP2 (1:1,000; cat. no. 5738; Cell Signaling Technology, Inc., Danvers, MA, USA), E-cadherin (1:1,000; cat. no. sc-71009; Santa Cruz Biotechnology, Inc., Santa Cruz, CA, USA), N-cadherin (1:1,000; cat. no. 4061; Cell Signaling Technology, Inc.) and GAPDH (1:1,500; cat. no. sc-51631; Santa Cruz Biotechnology, Inc.) overnight at $4^{\circ} \mathrm{C}$. Then, secondary antibodies (1:3,000; cat. no. sc-3744 and cat. no. sc-2089; Santa Cruz Biotechnology, Inc.) were incubated with membranes at room temperature for $2 \mathrm{~h}$. The expression level of detected proteins was visualized using ECL reagents (Amersham Biosciences Corp., Piscataway, NJ, USA).

Transwell assays. The migratory and invasive abilities of OC were evaluated by Transwell assay. The day prior to the Transwell assay, OC cells were starved in serum-free DMEM media overnight. After trypsinization, OC cells $\left(5 \times 10^{4}\right)$ were re-suspended in serum-free DMEM and seeded in the upper chamber of the Transwell inserts (Millipore, Billerica, MA, USA). The lower chamber of the Transwell inserts were filled with $700 \mu 1$ serum-containing DMEM (20\% FBS) as a chemoattractant. For the invasion assays, the upper Transwell chamber was coated with $100 \mu 1$ Matrigel (diluted in DMEM at the ratio of 1:6). Twenty-four hours later, the cells which did not migrate or invade through the membrane were removed with cotton swab while the cells that had migrated or invaded through the membrane were stained with crystal violet. The number of migrated or invaded OC cells was counted under light microscope.

Immunohistochemical (IHC) staining. To investigate the relationship between the expression of miR-616 and E-cadherin, $\mathrm{N}$-cadherin and TIMP2, 10 randomly selected OC tissues with a low miR-616 level and 10 OC tissues with a high miR-616 level were used to perform IHC staining. The paraformaldehydefixed clinical tissues were subjected to IHC staining. Paraffin sections (4- $\mu \mathrm{m}$ thickness) were subjected to deparaffinization and re-hydration through xylene and graded ethanol. These slides were incubated with $3 \%$ hydrogen peroxide for $10 \mathrm{~min}$ to quench the endogenous peroxidase activity. Then, the slides were blocked with goat serum at room temperature for $1 \mathrm{~h}$ and incubated with the E-cadherin (1:100; cat. no. sc-71009), $\mathrm{N}$-cadherin (1:100; cat. no. 4061$)$ or TIMP2 antibodies (1:50; cat. no. 5738$)$ at $4^{\circ} \mathrm{C}$ overnight. Secondary antibodies (cat. no. SAP-9101; ZSGB-Bio, Beijing, China) were then incubated with these slides at room temperature for $1 \mathrm{~h}$. Finally, these sections were stained with diaminobenzidine, and then hematoxylin. The intensity of IHC staining was classified into 4 grades: 0 , none; 1 , weak; 2 , moderate; and 3, strong. The percentage of positive staining was divided into the 5 grades: $0(<10 \%), 1(10-30 \%), 2$ (30-50\%), $3(51-70 \%)$ and $4(>70 \%)$. IHC scores were calculated by multiplying the percentage of positive cells $(\mathrm{P})$ by the intensity.

Luciferase reporter assay. A luciferase reporter assay was performed to investigate whether miR-616 interacted with the 3'-UTR of TIMP2. The binding sites of miR-616 within the TIMP2 3'-UTR construct in antisense orientation was further mutated using Q5 Site Directed Mutagenesis kit (New England Biolabs, Beverly, MA, USA). OC cells were seeded in 24-well plates at the density of $1-3 \times 10^{5}$ cells/well. Then, the cells cultured in 24-well plates were co-transfected with miR-616 mimics or miR-616 inhibitors along with the wild-type 3'-UTR of TIMP2 or the mutated 3'-UTR of TIMP2, and pRL-SV40 Renilla plasmid (Promega Corp., Madison, WI, USA). Cell transfection was performed using Lipofectamine ${ }^{\circledR} 2000$. Forty-eight hours later, the luciferase activities were evaluated using the Dual-Luciferase Reporter Assay system (Promega, Shanghai, China). 

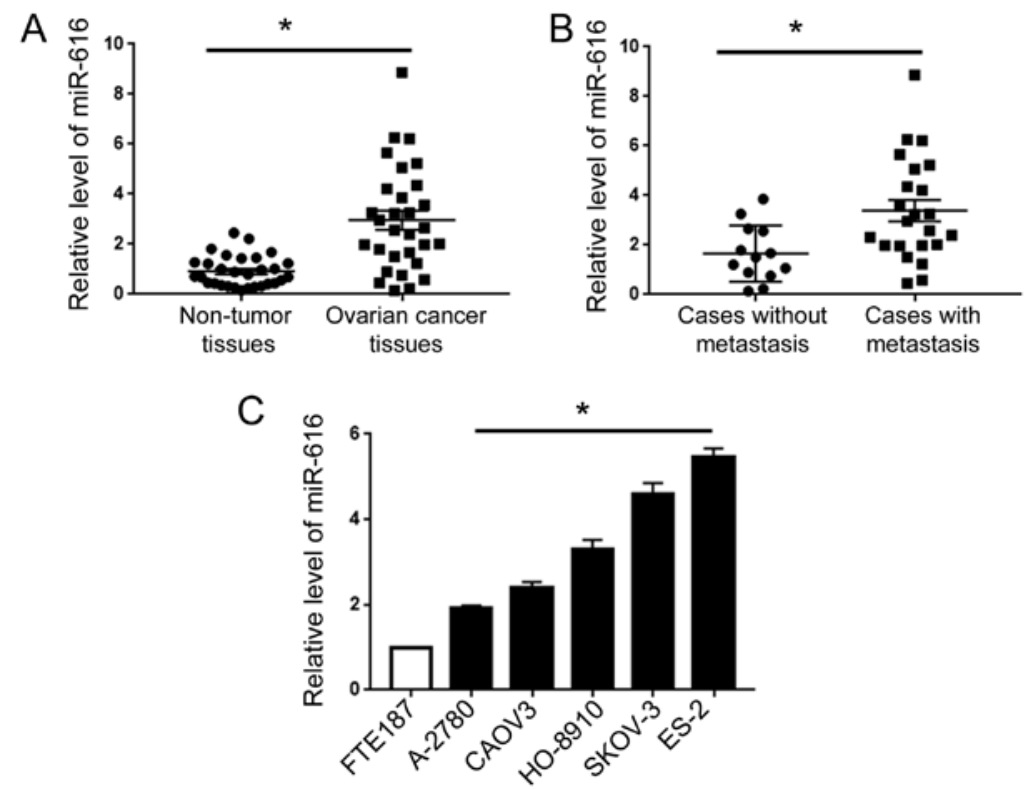

Figure 1. Expression of miR-616 in OC tissues and cells. (A) The expression level of miR-616 was compared between OC tissues and adjacent non-tumor tissues. (B) The expression level of miR-616 was compared between patients with and without metastasis. (C) The expression of miR-616 was evaluated in 5 OC cell lines (A2780, CAOV3, HO-8910, SKOV-3 and ES-2) and immortalized human fallopian tube epithelial cell line FTE187 cells. "P<0.05. OC, ovarian cancer.

In vivo experiments. Tail vein injection experiments were performed in nude mice to evaluate the in vivo metastatic capacity of OC cells. OC cells $\left(1 \times 10^{5}\right)$ transfected with control vector or miR-616 mimics, or, those transfected with negative control vector or miR-616 inhibitors, were injected into nude mice through tail veins. Eight weeks after tail vein injection, the mice were sacrificed and the lungs were isolated for hematoxylin and eosin (H\&E) staining. The protocols regarding the in vivo manipulations were approved by the Animal Care Committee of Zhejiang Cancer Hospital.

Statistical analysis. All data were expressed as the mean \pm standard error of the mean (SEM) and GraphPad Prism 5.0 (GraphPad Software, Inc., La Jolla, CA, USA) was used for statistical analysis in this study. After dividing OC patients into two groups based on the cut-off value defined as the median level of miR-616, differences of the Kaplan-Meier curves between the miR-616-high group and miR-616-low group were detected using the log-rank test. $\mathrm{P}<0.05$ was considered to indicate a statistically significant result.

\section{Results}

miR-616 is upregulated in OC tissues and cell lines. qRT-PCR for clinical tissues revealed that compared with adjacent non-tumor tissues, OC tissues exhibited an increased level of miR-616 ( $\mathrm{P}<0.05$; Fig. 1A). Then, we compared the level of miR-616 in patients with or without metastasis. Compared with the patients without metastasis, patients with metastasis exhibited an elevated level of miR-616 ( $\mathrm{P}<0.05 ;$ Fig. 1B). Additionally, we assessed the expression level of miR-616 in human fallopian tube epithelial cells (FTE187 cells) and five types of human OC cells (A2780, CAOV3, HO-8910, SKOV-3 and ES-2). Compared with the FTE187 cells, all five OC cell lines exhibited an increased level of miR-616, with the highest level in ES-2 cells and the lowest level in A-2780 cells $(\mathrm{P}<0.05$; Fig. 1C).
Table I. Association analysis between the expression level of miR-616 and the clinical features of OC patients.

\begin{tabular}{|c|c|c|c|c|}
\hline & $\begin{array}{c}\text { miR-616 } \\
\text { expression level }\end{array}$ & & & \\
\hline Clinical features & No. of patients & Low & High & $\mathrm{P}$-value \\
\hline \multicolumn{5}{|l|}{ Age (years) } \\
\hline$<50$ & 25 & 15 & 10 & 0.295 \\
\hline$\geq 50$ & 35 & 15 & 20 & \\
\hline \multicolumn{5}{|l|}{ Histological type } \\
\hline High-grade serous & 36 & 20 & 16 & 0.516 \\
\hline Clear cell & 12 & 5 & 7 & \\
\hline Endometrioid & 7 & 4 & 3 & \\
\hline Mucinous & 3 & 1 & 2 & \\
\hline Low-grade serous & 2 & 0 & 2 & \\
\hline \multicolumn{5}{|l|}{ Tumor differentiation } \\
\hline Low & 33 & 12 & 21 & 0.048 \\
\hline Moderate & 15 & 9 & 6 & \\
\hline High & 12 & 9 & 3 & \\
\hline \multicolumn{5}{|l|}{ TNM stage } \\
\hline I-II & 24 & 17 & 7 & 0.017 \\
\hline III-IV & 36 & 13 & 23 & \\
\hline
\end{tabular}

An increased miR-616 level confers poor clinical features and prognosis of OC patients. We further investigated the prognostic significance of miR-616 in OC. OC patients were divided into two groups based on the cut-off value defined as the median level of miR-616: low miR-616 group $(n=30)$ and high miR-616 group $(n=30)$. Association analysis revealed that a high miR-616 level was associated with poor tumor differentiation $(\mathrm{P}=0.048)$ and advanced TNM stage $(\mathrm{P}=0.017)$ (Table I). Kaplan-Meier 

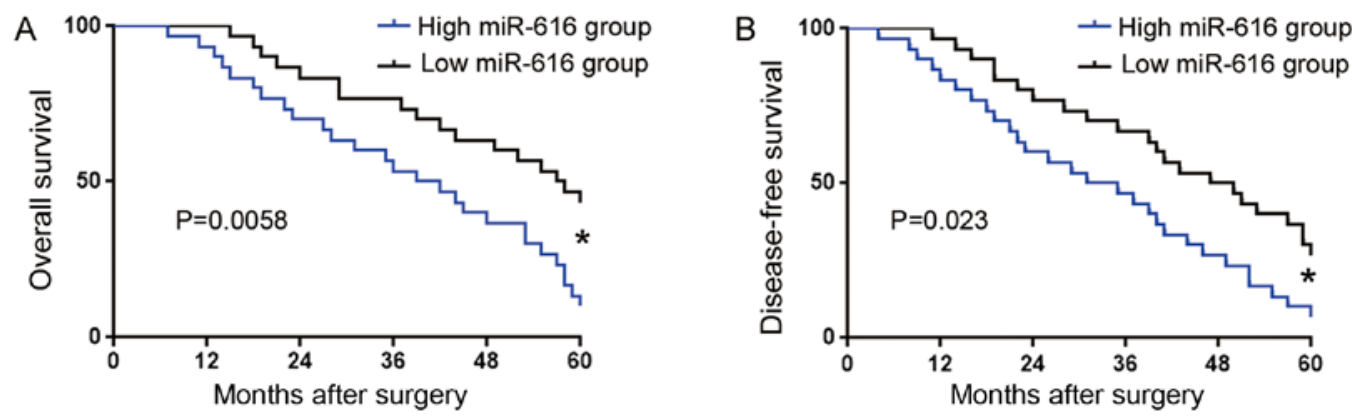

Figure 2. The prognostic value of miR-616 in OC patients. (A) Overall and (B) disease-free survival was compared between patients with a high miR-616 level and those with a low miR-616 level. ${ }^{\mathrm{P}}<0.05$. OC, ovarian cancer.

A

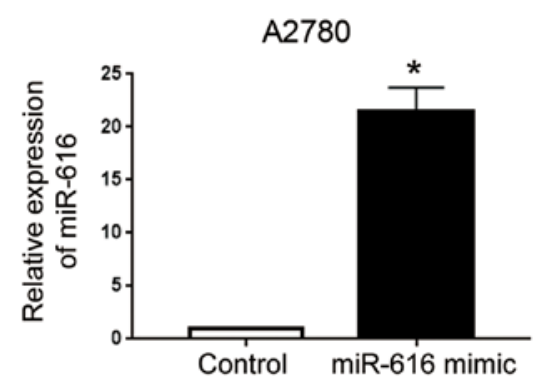

C

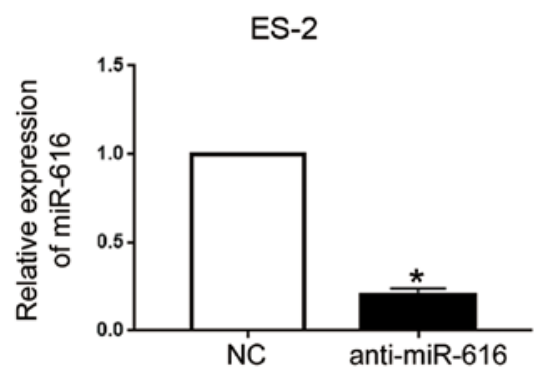

B Control A2780

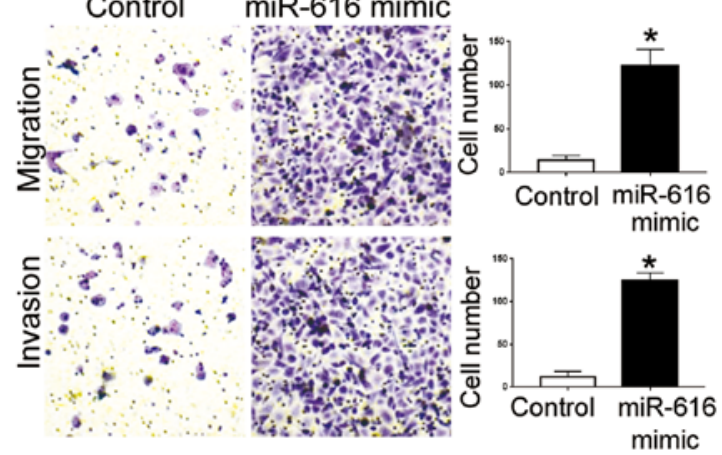

D

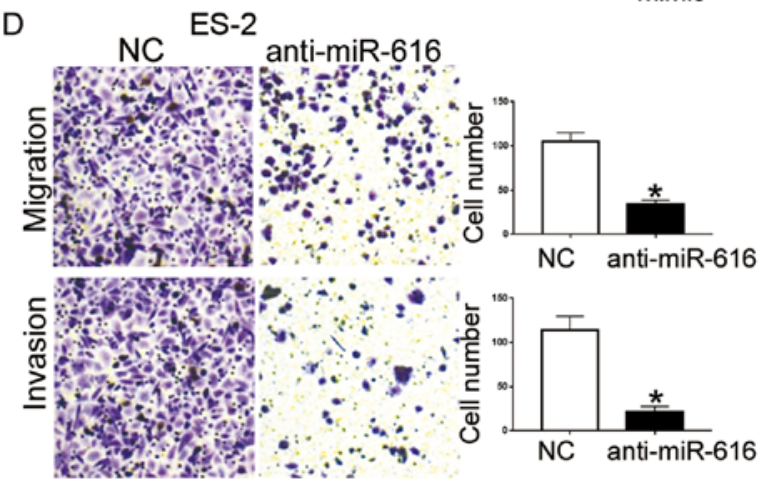

Figure 3. miR-616 promotes the migration and invasion of OC cells. (A) Transfection of miR-616 mimics significantly increased the expression level of miR-616 in A2780 cells. (B) miR-616 overexpression increased the migration and invasion of A2780 cells. (C) Transfection of miR-616 inhibitors significantly decreased the expression level of miR-616 in ES-2 cells. (D) miR-616 knockdown decreased the migration and invasion of ES-2 cells. "P<0.05. OC, ovarian cancer.

analysis (Fig. 2) revealed that compared with those in the low miR-616 group, patients with a high miR-616 level had significantly decreased overall survival (OS) and disease-free survival (DFS). These results revealed that the expression level of miR-616 could indicate the unfavorable clinical features and poor prognosis of $\mathrm{OC}$ patients.

miR-616 potentiates the metastatic ability of OC cells. Next, we transfected A2780 cells with miR-616 mimics to overexpress miR-616, and, transfected ES-2 cells with miR-616 inhibitors to knockdown miR-616. Compared with the control vector, transfection of miR-616 mimics led to a significantly increased miR-616 level in A2780 cells ( $\mathrm{P}<0.05$; Fig. 3A). Transwell assays further revealed that forced miR-616 expression in A2780 cells led to increased migration and invasion of A2780 cells $(\mathrm{P}<0.05$; Fig. 3B). Conversely, transfection of miR-616 inhibitors effectively knocked down miR-616 in ES-2 cells $(\mathrm{P}<0.05$; Fig. $3 \mathrm{C}$ ), and subsequently resulted in reduced migration and invasion of ES-2 cells $(\mathrm{P}<0.05$; Fig. 3D).

miR-616 promotes the EMT of OC cells. Since EMT has been widely accepted as an important mechanism of cancer metastasis (12-14), we further investigated whether miR-616 regulated EMT of OC cells. Overexpression of miR-616 in A2780 cells decreased the level of E-cadherin and increased the level of $\mathrm{N}$-cadherin $(\mathrm{P}<0.05$; Fig. 4A). Conversely, knockdown of miR-616 in ES-2 cells led to increased E-cadherin expression and decreased $\mathrm{N}$-cadherin expression $(\mathrm{P}<0.05$; Fig. 4B). Furthermore, we randomly selected 10 OC tissues with low miR-616 levels and 10 OC tissues with high miR-616 levels to perform IHC staining for E-cadherin and N-cadherin, and compared their expression level in OC tissues with low and high miR-616 levels. Compared with the tissues expressing low miR-616, tissues expressing high miR-616 exhibited decreased 

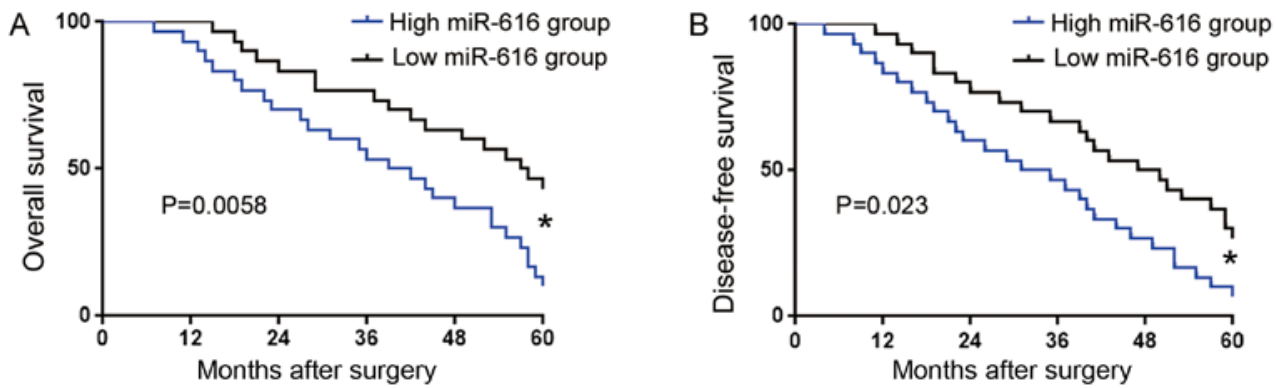

Figure 4. miR-616 promotes the EMT of OC cells. (A) Western blotting for E-cadherin and N-cadherin in A2780 cells transfected with control mimics or miR-616 mimics. miR-616 overexpression decreased E-cadherin expression and increased N-cadherin expression. (B) Western blotting for E-cadherin and N-cadherin in ES-2 cells transfected with negative control inhibitors or miR-616 inhibitors. miR-616 knockdown increased E-cadherin expression and decreased N-cadherin expression. (C) IHC staining for E-cadherin and N-cadherin in OS tissues with a low miR-616 expression level and those with a high miR-616 expression level. ${ }^{*} \mathrm{P}<0.05$. EMT, epithelial-mesenchymal transition; OC, ovarian cancer; IHC, immunohistochemical.
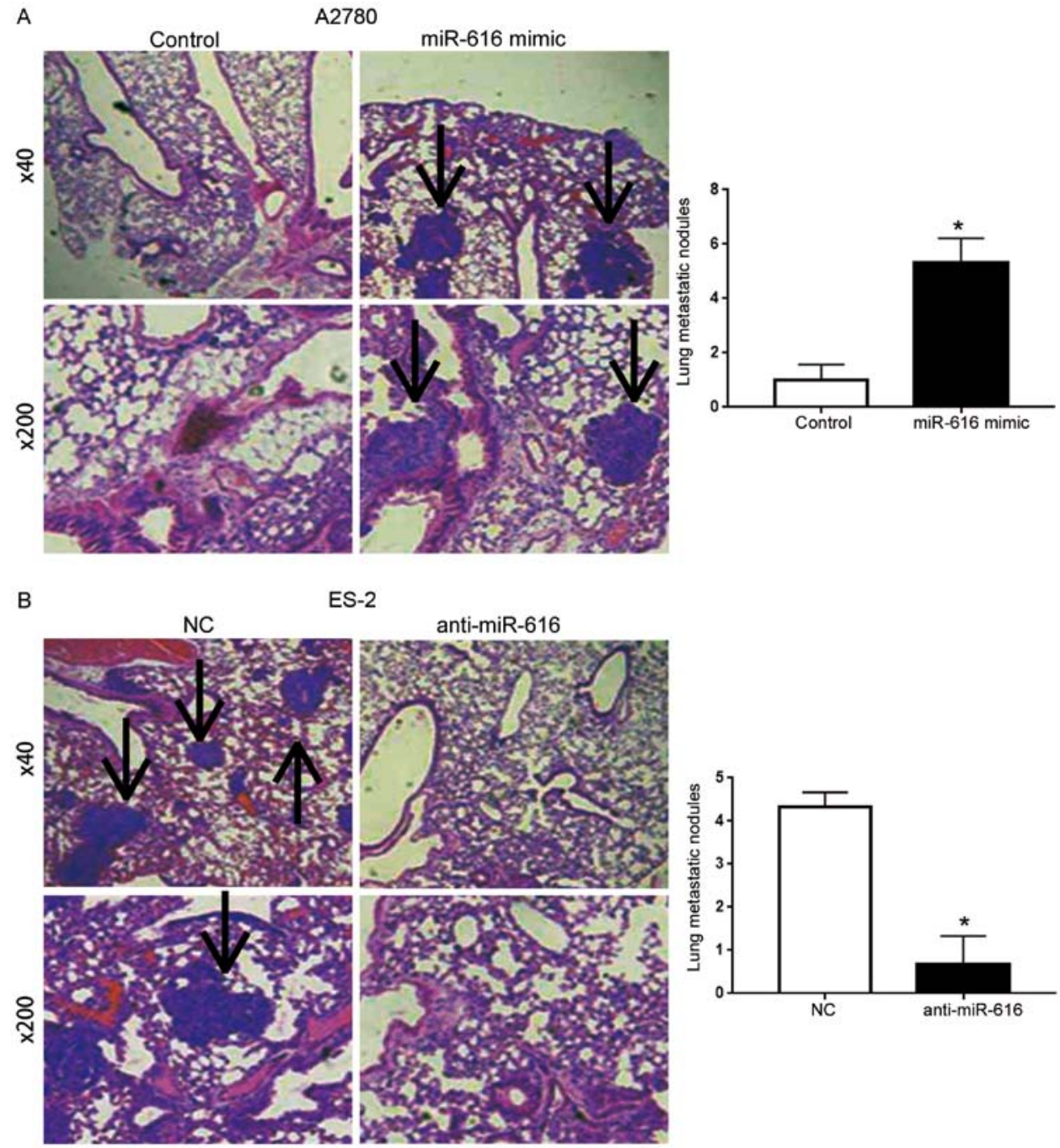

Figure 5. miR-616 potentiates the metastatic ability of OC cells in vivo. (A) Overexpression of miR-616 increased the lung metastasis of A2780 cells in nude mice. (B) Knockdown of miR-616 decreased the lung metastasis of ES-2 cells in nude mice. "P $<0.05$. OC, ovarian cancer.

E-cadherin level and increased $\mathrm{N}$-cadherin level $(\mathrm{P}<0.05$; Fig. 4C). Collectively, these data indicated that miR-616 promoted the EMT of OC cells.

miR-616 promotes the lung metastasis of OC cells in nude mice. To further elucidate the influence of miR-616 on the in vivo metastatic ability of OC cells, we performed a tail vein injection assay using A2780 cells overexpressing miR-616 and ES-2 cells with miR-616 knockdown. Compared with the A2780 cells in the control group, the tail vein injection of A2780 cells overexpressing miR-616 resulted in an increased number of lung metastatic nodules $(\mathrm{P}<0.05$; Fig. 5A). Knockdown of miR-616 in ES-2 cells led to a decreased number of lung metastasis nodules $(\mathrm{P}<0.05$; Fig. 5B). 


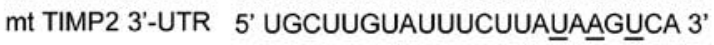

B
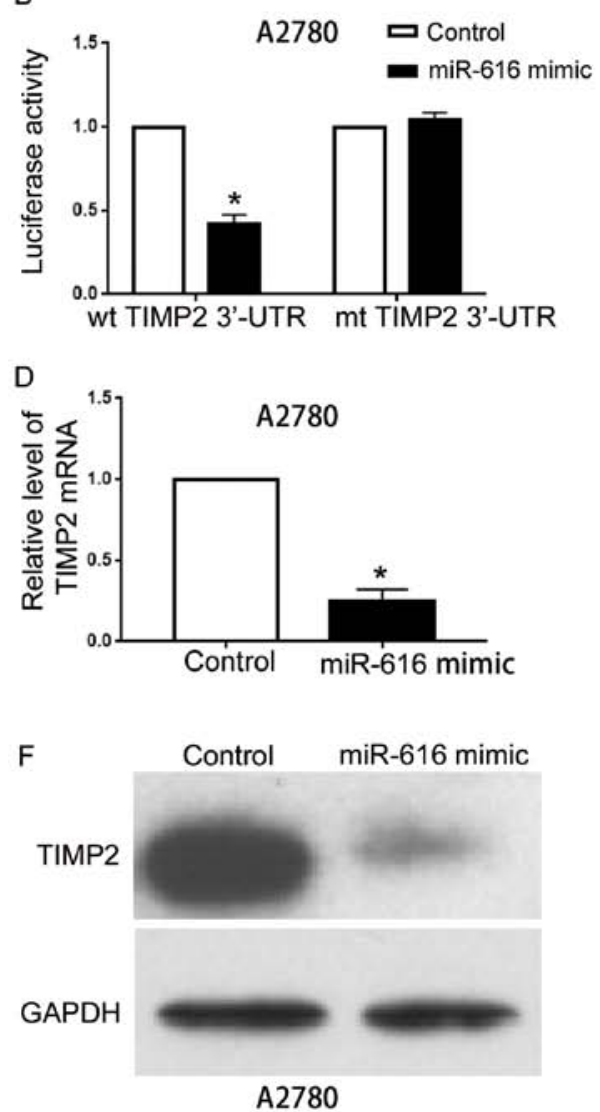

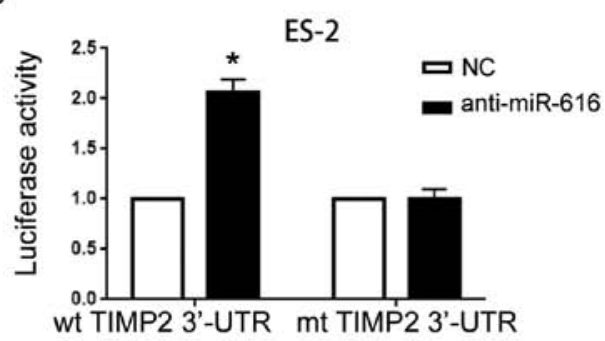

E

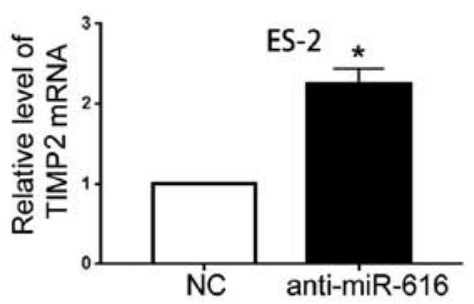

G

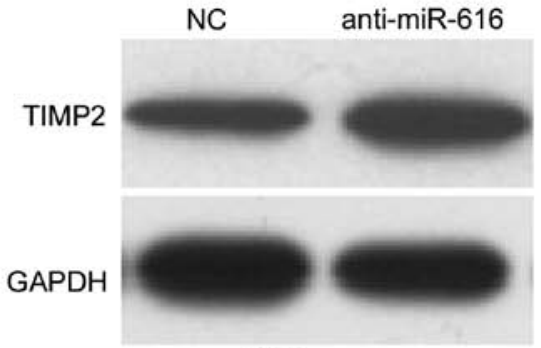

ES-2
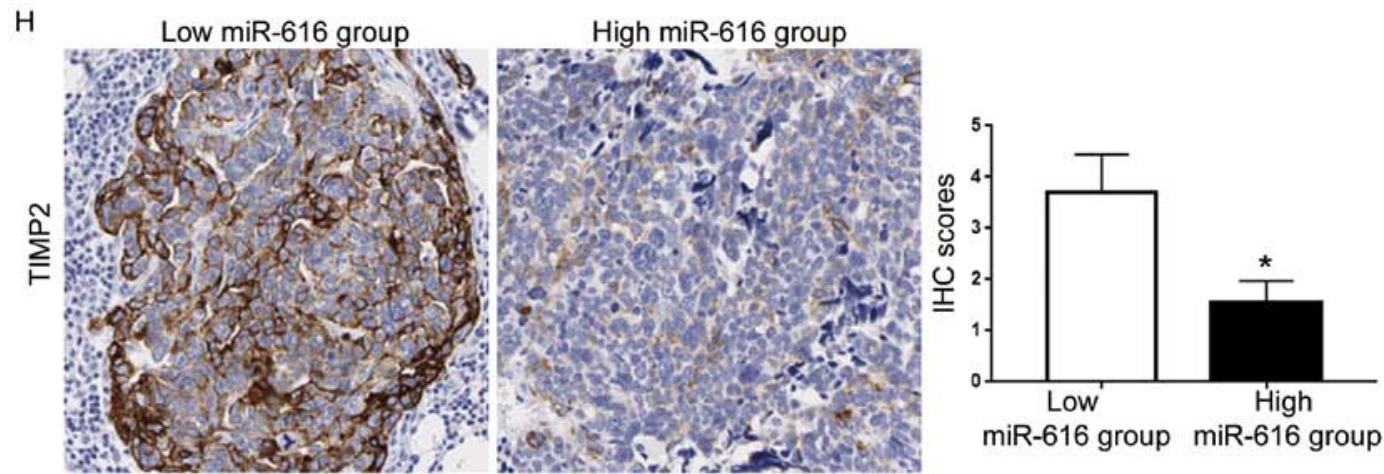

Figure 6. TIMP2 is a direct target of miR-616 in OC. (A) miR-616 contained the complementary sequence mediating the binding of miR-616 with TIMP2 3'-UTR. (B) Overexpression of miR-616 decreased the luciferase activity of the wild-type (wt) TIMP2 3'-UTR but had no obvious influence on that of the mutated (mt) TIMP2 3'-UTR. (C) Inhibition of miR-616 significantly increased the luciferase activity of the wt 3'-UTR of TIMP2 but had no obvious influence on that of the mt 3'-UTR of TIMP2. (D) Overexpression of miR-616 significantly decreased the mRNA level of TIMP2 in A2780 cells. (E) Inhibition of miR-616 increased the mRNA level of TIMP2 in ES-2 cells. (F) Overexpression of miR-616 significantly decreased the protein level of TIMP2 in A2780 cells. (G) Inhibition of miR-616 significantly increased the level of TIMP2 protein in ES-2 cells. (H) Immunohistochemical (IHC) staining for TIMP-2 in OC tissues with low miR-616 levels and those with high miR-616 levels. " $\mathrm{P}<0.05$. OC, ovarian cancer; TIMP2, tissue inhibitor of metalloproteinases 2.

$T I M P 2$ is a direct target of miR-616 in $O C$. After elucidating the expression and function of miR-616 in OC, we further investigated the mechanisms underlying the functions of miR-616 in OC. We searched the databases in the websites TargetScan 6.2 and miRanda to identify the potential target of miR-616. The data on these two websites revealed that miR-616 contained the complementary sequences mediating its interaction with TIMP2 3'-UTR (Fig. 6A). A luciferase activity assay revealed that miR-616 overexpression decreased the luciferase activity of the wild-type (wt) TIMP2 3'-UTR (P<0.05; Fig. 6B) without affecting that of the mutated (mt) TIMP2 3'-UTR (Fig. 6B). Conversely, miR-616 knockdown resulted in increased luciferase activity of the wt TIMP2 3'-UTR (P<0.05; Fig. 6C) and did not affect that of the mt TIMP2 3'-UTR (Fig. 6C). These results indicated that miR-616 interacts with TIMP2 3 '-UTR through complementary sequences. The results of qRT-PCR 
A
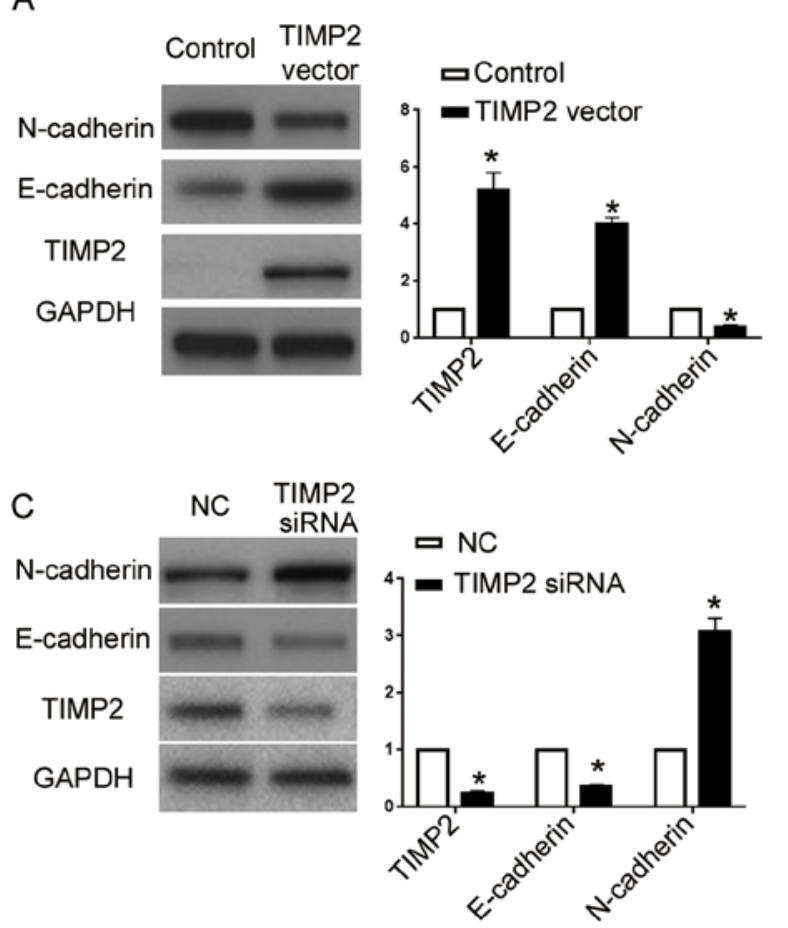
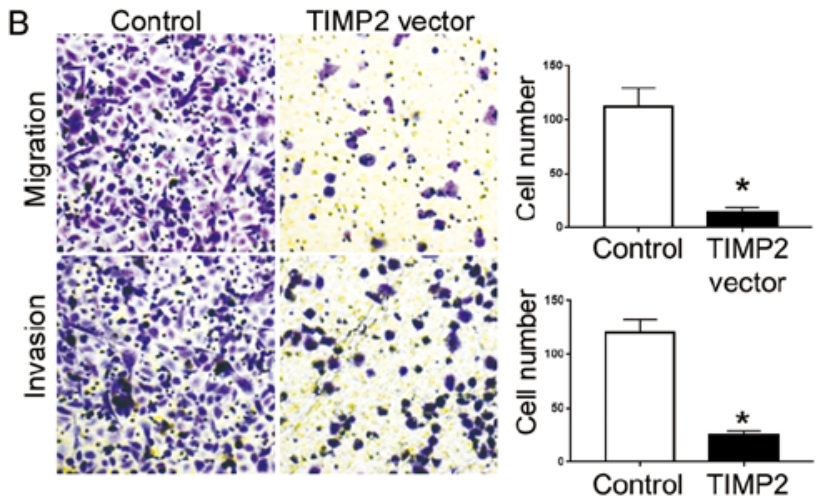

D
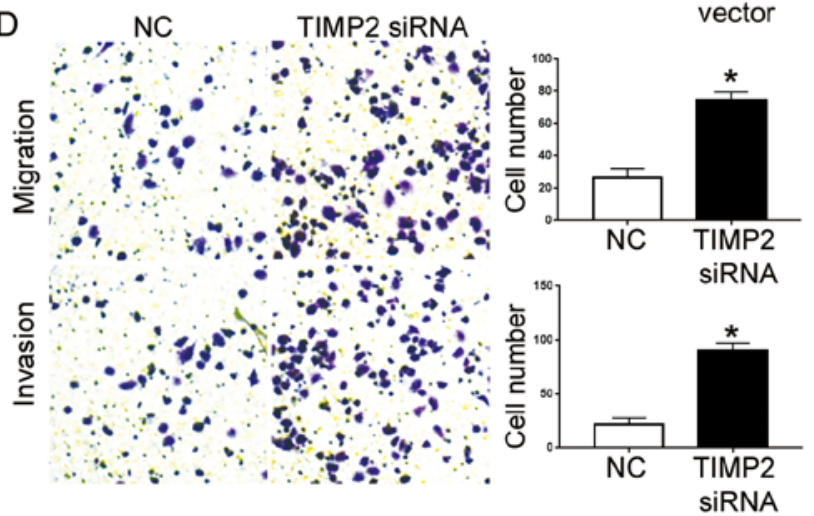

Figure 7. miR-616 exerts promoting effects on the metastasis of OC cells by targeting TIMP2. (A) A2780 cells overexpressing miR-616 were transfected with the control vector or TIMP2 vector. Transfection of the TIMP2 vector restored TIMP2 expression in A2780 cells overexpressing miR-616, and led to increased E-cadherin and decreased N-cadherin. (B) Restoration of TIMP2 inhibited the promoting effects of miR-616 overexpression on migration and invasion of A2780 cells. (C) ES-2 cells with miR-616 knockdown were transfected with negative control siRNA or TIMP2-siRNA. Transfection of TIMP2-siRNA decreased TIMP2 expression in ES-2 cells with miR-616 knockdown, and led to decreased E-cadherin expression and increased N-cadherin. (D) Knockdown of TIMP2 reversed the inhibitory effects of miR-616 knockdown on the migration and invasion of ES-2 cells. ${ }^{*}<<0.05$. OC, ovarian cancer; TIMP2, tissue inhibitor of metalloproteinases 2 .

and western blotting revealed that forced expression of miR-616 led to decreased mRNA and protein levels of TIMP2 in A2780 cells ( $\mathrm{P}<0.05$; Fig. 6D and F) while miR-616 knockdown led to increased mRNA and protein levels of TIMP2 in ES-2 cells $(\mathrm{P}<0.05$; Fig. $6 \mathrm{E}$ and $\mathrm{G})$. Furthermore, IHC in $\mathrm{OC}$ tissues revealed that compared with tissues expressing a low miR-616 level, tissues with a high miR-616 level exhibited a significantly decreased level of TIMP2 $(\mathrm{P}<0.05$; Fig. $6 \mathrm{H})$. Collectively, these data indicated that TIMP2 is a downstream target of miR-616 in OC.

miR-616 exerts the promoting effects on OC metastasis by inhibiting TIMP2 expression. Lastly, we overexpressed TIMP2 in A2780 cells with miR-616 overexpression. In the A2780 cells overexpressing miR-616, transfection of the TIMP2 vector significantly increased TIMP2 expression $(\mathrm{P}<0.05$; Fig. 7A), and resulted in increased E-cadherin expression and decreased $\mathrm{N}$-cadherin expression ( $\mathrm{P}<0.05$; Fig. 7A). Transwell assays revealed that overexpression of TIMP2 abrogated the effects of miR-616 overexpression on cell migration and invasion $(\mathrm{P}<0.05$; Fig. 7B). On the other hand, TIMP2siRNA significantly decreased TIMP2 expression in ES-2 cells with miR-616 knockdown $(\mathrm{P}<0.05$; Fig. 7C) and resulted in decreased E-cadherin and increased $\mathrm{N}$-cadherin $(\mathrm{P}<0.05$; Fig. 7C). Functionally, TIMP2 knockdown abrogated the inhibiting effects of miR-616 knockdown on cell metastatic ability $(\mathrm{P}<0.05$; Fig. 7D).

\section{Discussion}

Cancer metastasis is an important cause for the poor survival of cancer patients. The mechanisms underlying the metastatic processes of cancer cells are complex and remain largely unknown. Accumulating evidence has demonstrated that miRNAs play critical roles in cancer metastasis (15). miRNAs have been revealed to regulate the migration, invasion, extracellular matrix degradation and EMT of cancer cells, to facilitate the occurrence of cancer metastasis (16). Therefore, miRNAs have been demonstrated to be promising biomarkers and therapeutic targets of OC.

miR-616 was recently identified to be a cancer-associated miRNA. miR-616 was found to promote metastasis and EMT of HCC cells by targeting PTEN expression (9). miR-616 was demonstated to promote the growth of prostate cancer cells by inhibiting the expression of TFPI-2 (10). The latest research in non-small cell lung cancer revealed that miR-616 promoted the growth and metastasis by targeting SOX7. This present study revealed that miR-616 expression was increased in OC cells and tissues. An elevated miR-616 level in OC was associated with poor prognosis of OC patients. Forced expression of miR-616 increased the migration and invasion of A2780 cells while miR-616 knockdown inhibited the metastasis of ES-2 cells. In vivo experiments in nude mice revealed that miR-616 overexpression increased the lung metastasis of A2780 cells while miR-616 knockdown decreased the lung metastasis of ES-2 
cells. These results indicated that miR-616 plays oncogenic roles in OC by enhancing cell metastasis both in vitro and in vivo.

EMT, characterized as a reduction of epithelial marker (E-cadherin) expression and an increase of mesenchymal marker (N-cadherin) expression (17), is an important cause for the enhanced ability of cancer metastasis (18). In this study, miR-616 overexpression was found to promote EMT of A2780 cells as suggested by decreased E-cadherin expression and increased N-cadherin expression. In contrast, miR-616 knockdown inhibited EMT of ES-2 cells. Data in OS tissues revealed that compared with tissues with low miR-616 expression, tissues with high miR-616 levels exhibited decreased E-cadherin expression and increased $\mathrm{N}$-cadherin expression. These data demonstrated that miR-616 enhanced the metastasis of OC cells by promoting EMT.

Tissue inhibitor of metalloproteinase (TIMPs) are a group of proteins inhibiting the activity of matrix metalloproteinases (MMPs) $(19,20)$. TIMP-2 belongs to the TIMP family and inhibits MMP-2 activity (21). It has been revealed to inhibit the angiogenesis and growth of cancer cells (22). A study on pancreatic cancer revealed that TIMP2 was involved in the metastasis and EMT of cancer cells regulated by miR-106a (23). In the present study, we found that miR-616 interacted with TIMP2 3'-UTR and inhibited the expression of TIMP2 in OC cells. Notably, tissues expressing a high miR-616 level exhibited decreased expression of TIMP2. Furthermore, we demonstrated that TIMP2 was involved in the promoting effects of miR-616 on cell migration, invasion and EMT. Restoring TIMP2 expression reduced the effects of miR-616 overexpression on the metastasis and EMT of A2780 cells. Conversely, knockdown of TIMP2 abrogated the inhibiting effects of miR-616 knockdown on the metastasis and EMT of ES-2 cells.

In conclusion, out results revealed that the expression level of miR-616 is elevated in OC. A high miR-616 level was associated with unfavorable clinical features and poor prognosis of OC patients. miR-616 promoted the migration, invasion and EMT of OC cells. Animal experiments demonstrated that miR-616 enhanced the occurrence of lung metastasis of OC cells in nude mice. Furthermore, TIMP2 was identified to be the downstream target of miR-616. Inhibition of TIMP2 expression was critical for the promoting effects of miR-616 on cell migration, invasion and EMT. This study demonstrated that miR-616 is an oncogenic miRNA in OC and promotes the progression of OC by enhancing the metastatic ability of OC cells.

\section{Acknowledgements}

Not applicable.

\section{Funding}

The present study was supported by the Zhejiang Medical and Health Research fund (no. 2017KY248) and the Zhejiang Traditional Chinese Medicine Research fund (no. 2017ZA035).

\section{Availability of data and materials}

The datasets used duing the present study are available from the corresponding author upon reasonable request.

\section{Authors' contributions}

ZBC conceived and designed this study, ZBC and JQZ performed the in vitro and in vivo experiments, $\mathrm{YMZ}$ performed the data analysis, ZBC and JJW wrote and revised the manuscript. All authors read and approved the manuscript and agree to be accountable for all aspects of the research in ensuring that the accuracy or integrity of any part of the work are appropriately investigated and resolved.

\section{Ethics approval and consent to participate}

The protocol of this study was approved by the Institutional Research Ethics Committee of Zhejiang Cancer Hospital and informed consent was obtained from every patient enrolled in this study. The protocols regarding the in vivo manipulations were approved by the Animal Care Committee of Zhejiang Cancer Hospital.

\section{Consent for publication}

Not applicable

\section{Competing interests}

The authors state that they have no competing interests.

\section{References}

1. Bast RC Jr, Hennessy B and Mills GB: The biology of ovarian cancer: New opportunities for translation. Nat Rev Cancer 9: 415-428, 2009.

2. Holschneider $\mathrm{CH}$ and Berek JS: Ovarian cancer: Epidemiology, biology, and prognostic factors. Semin Surg Oncol 19: 3-10, 2000.

3. Cai Y, Yu X, Hu S and Yu J: A brief review on the mechanisms of miRNA regulation. Genomics Proteomics Bioinformatics 7: 147-154, 2009.

4. Yates LA, Norbury CJ and Gilbert RJ: The long and short of microRNA. Cell 153: 516-519, 2013.

5. Alvarez-Garcia I and Miska EA: MicroRNA functions in animal development and human disease. Development 132: 4653-4662, 2005.

6. Farazi TA, Hoell JI, Morozov P and Tuschl T: MicroRNAs in human cancer. In: MicroRNA Cancer Regulation. Volume 774 Schmitz U, Wolkenhauer O and Vera J (eds). Springer, Dordrecht, ppl-20, 2013.

7. Calin GA and Croce CM: MicroRNA signatures in human cancers. Nat Rev Cancer 6: 857-866, 2006.

8. Iorio MV, Visone R, Di Leva G, Donati V, Petrocca F, Casalini P, Taccioli C, Volinia S, Liu CG, Alder H, et al: MicroRNA signatures in human ovarian cancer. Cancer Res 67: 8699-8707, 2007.

9. Zhang D, Zhou P, Wang W, Wang X, Li J, Sun X and Zhang L: MicroRNA-616 promotes the migration, invasion and epithelialmesenchymal transition of HCC by targeting PTEN. Oncol Rep 35: 366-374, 2016.

10. Ma S, Chan YP, Kwan PS, Lee TK, Yan M, Tang KH, Ling MT, Vielkind JR, Guan XY and Chan KW: MicroRNA-616 induces androgen-independent growth of prostate cancer cells by suppressing expression of tissue factor pathway inhibitor TFPI-2. Cancer Res 71: 583-592, 2011.

11. Wang DX, Zou YJ, Zhuang XB, Chen SX, Lin Y, Li WL, Lin JJ and Lin ZQ: Sulforaphane suppresses EMT and metastasis in human lung cancer through miR-616-5p-mediated GSK3 $\beta /$ $\beta$-catenin signaling pathways. Acta Pharmacol Sin 38: 241-251, 2017.

12. Yilmaz M and Christofori G: EMT, the cytoskeleton, and cancer cell invasion. Cancer Metastasis Rev 28: 15-33, 2009.

13. Moreno-Bueno G, Portillo F and Cano A: Transcriptional regulation of cell polarity in EMT and cancer. Oncogene 27: 6958-6969, 2008. 
14. Sánchez-Tilló E, Liu Y, de Barrios O, Siles L, Fanlo L, Cuatrecasas M, Darling DS, Dean DC, Castells A and Postigo A: EMT-activating transcription factors in cancer: Beyond EMT and tumor invasiveness. Cell Mol Life Sci 69: 3429-3456, 2012.

15. Baranwal S and Alahari SK: miRNA control of tumor cell invasion and metastasis. Int J Cancer 126: 1283-1290, 2010.

16. De Craene B and Berx G: Regulatory networks defining EMT during cancer initiation and progression. Nat Rev Cancer 13 97-110, 2013

17. Kalluri R: EMT: When epithelial cells decide to become mesenchymal-like cells. J Clin Invest 119: 1417-1419, 2009.

18. Gotzmann J, Mikula M, Eger A, Schulte-Hermann R, Foisner R, Beug $\mathrm{H}$ and Mikulits W: Molecular aspects of epithelial cell plasticity: Implications for local tumor invasion and metastasis. Mutat Res 566: 9-20, 2004
19. Egeblad $\mathrm{M}$ and Werb Z: New functions for the matrix metalloproteinases in cancer progression. Nat Rev Cancer 2: 161-174, 2002.

20. Vynios DH: TIMP2 (TIMP metallopeptidase inhibitor 2). Atlas Genet Cytogenet Oncol Haematol 13: 229-231, 2009.

21. Nakopoulou L, Tsirmpa I, Alexandrou P, Louvrou A, Ampela C, Markaki S and Davaris PS: MMP-2 protein in invasive breast cancer and the impact of MMP-2/TIMP-2 phenotype on overall survival. Breast Cancer Res Treat 77: 145-155, 2003.

22. Duffy MJ, McGowan PM and Gallagher WM: Cancer invasion and metastasis: Changing views. J Pathol 214: 283-293, 2008.

23. Li P, Xu Q, Zhang D, Li X, Han L, Lei J, Duan W, Ma Q, Wu Z and Wang Z: Upregulated miR-106a plays an oncogenic role in pancreatic cancer. FEBS Lett 588: 705-712, 2014. 